\title{
CHANGING THE PARADIGM FOR MANAGEMENT OF PEDIATRIC PRIMARY SPONTANEOUS PNEUMOTHORAX: A SIMPLE ASPIRATION TEST PREDICTS NEED FOR OPERATION
}

Charles M. Leys, MD, MSCI ${ }^{1}$; Ronald B. Hirschl, MD²; Jonathan E. Kohler, MD ${ }^{1}$; Linda CherneyStafford $^{1}$; Nicholas Marka, MS ${ }^{1}$; Mary E. Fallat, MD $^{3}$; Samir K. Gadepalli, MD, MBA ${ }^{2}$; Jason D. Fraser, $\mathrm{MD}^{4}$; Julia Grabowski, MD ${ }^{5}$; R. Cartland Burns, MD ${ }^{6}$, Cynthia D. Downard, MD ${ }^{3}$; David S. Foley, MD $^{3}$; Devin R. Halleran, $\mathrm{MD}^{7}$, Michael A. Helmrath, $\mathrm{MD}^{8}$; Rashmi Kabre, MD ${ }^{5}$; Michelle S. Knezevich, MS ${ }^{9}$, Dave R. Lal, MD ${ }^{9}$; Matthew P. Landman, MD, MPH ${ }^{6}$; Amy E. Lawrence, MD ${ }^{7}$, Grace Z. Mak, MD ${ }^{10}$; Peter C. Minneci, MD MHSc ${ }^{7}$; Ninette Musili ${ }^{2}$; Beth Rymeski, DO ${ }^{8}$; Jacqueline M. Saito MD $^{11}$; Thomas T. Sato, MD ${ }^{9}$; Shawn D. St. Peter, MD ${ }^{4}$; Brad W. Warner, MD ${ }^{11}$; Daniel J. Ostlie, $\mathrm{MD}^{12}$; on behalf of the Midwest Pediatric Surgery Consortium (MWPSC)

1) Division of Pediatric Surgery, Department of Surgery, University of Wisconsin School of Medicine and Public Health, Madison, WI

2) Division of Pediatric Surgery, Department of Surgery, University of Michigan School of Medicine, Ann Arbor, MI

3) Division of Pediatric Surgery, Department of Surgery, University of Louisville School of Medicine, Louisville, KY

4) Division of Pediatric Surgery, Department of Surgery, Children's Mercy Hospital, Kansas City, MO

5) Division of Pediatric Surgery, Department of Surgery, Northwestern University Feinberg School of Medicine, Chicago, IL

6) Division of Pediatric Surgery, Department of Surgery, Indiana University School of Medicine, Indianapolis, IN

7) Center for Surgical Outcomes Research, Department of Pediatric Surgery, Nationwide Children's Hospital, Columbus, $\mathrm{OH}$

8) Division of Pediatric Surgery, Department of Surgery, University of Cincinnati College of Medicine, Cincinnati, $\mathrm{OH}$

9) Division of Pediatric Surgery, Department of Surgery, Medical College of Wisconsin, Milwaukee, WI

10) Section of Pediatric Surgery, Department of Surgery, The University of Chicago Medicine and Biologic Sciences, Chicago, IL

11) Division of Pediatric Surgery, Department of Surgery, Washington University School of Medicine, St. Louis, MO

12) Department of Surgery, Phoenix Children's Hospital, Phoenix, AZ

\author{
Corresponding Author: \\ Charles M. Leys, MD, MSCI \\ Division of Pediatric Surgery \\ University of Wisconsin School of Medicine and Public Health \\ 600 Highland Ave, H4/740 \\ Madison, WI. 53792-7375 \\ Telephone: (608) 262-0466 \\ Fax: (608) 261-1876 \\ E-mail: Leys@ surgery.wisc.edu
}

This is the author's manuscript of the article published in final edited form as:

Leys, C. M., Hirschl, R. B., Kohler, J. E., Cherney-Stafford, L., Marka, N., Fallat, M. E., .. Ostlie, D. J. (2019). Changing the Paradigm for Management of Pediatric Primary Spontaneous Pneumothorax: A Simple Aspiration Test Predicts Need for Operation. Journal of Pediatric Surgery. https://doi.org/10.1016/j.jpedsurg.2019.09.043 
How this paper will improve care: Simple aspiration as first line intervention for spontaneous pneumothorax can identify patients with higher likelihood of prolonged air leak or recurrence necessitating surgery. The presented algorithm incorporating simple aspiration may be useful to support an earlier decision for surgical intervention.

\section{ABSTRACT:}

Purpose: Chest tube (CT) management for pediatric primary spontaneous pneumothorax (PSP) is associated with long hospital stays and high recurrence rates. To streamline management, we explored simple aspiration as a test to predict need for surgery.

Methods: A multi-institution, prospective pilot study of patients with first presentation for PSP at 9 children's hospitals was performed. Aspiration was performed through a pigtail catheter, followed by 6 hours observation with CT clamped. If pneumothorax recurred during observation, the aspiration test failed and subsequent management per surgeon discretion.

Results: Thirty-three patients were managed with simple aspiration. Aspiration was successful in 16 of $33(48 \%)$, while $17(52 \%)$ failed the aspiration test and required hospitalization. Twelve who failed aspiration underwent CT management, of which 10 (83\%) failed CT management due to either persistent air leak requiring VATS or subsequent PSP recurrence. Recurrence rate was significantly greater in the group that failed aspiration compared to the group that passed aspiration [10/12 (83\%) vs 7/16 (44\%), respectively, $\mathrm{P}=0.028]$.

Conclusion: Simple aspiration test upon presentation with PSP predicts chest tube failure with $83 \%$ positive predictive value. We recommend changing the PSP management algorithm to include an initial simple aspiration test, and if that fails, proceed directly to VATS. 
Keywords: Spontaneous pneumothorax; Simple aspiration; VATS; Thoracoscopic blebectomy

Type of Study: Prospective Pilot Study

Level of Evidence: Level III 


\section{INTRODUCTION}

The optimal initial management of a child with first presentation for primary spontaneous pneumothorax (PSP) remains unclear. The lack of an evidence-based pediatric-specific guideline has resulted in significant variability among pediatric surgeons.[1] A recent survey reported that the majority of North American pediatric surgeons perform chest tube placement for a first episode PSP, reserving surgery for recurrence or prolonged persistent air leak.[1] This approach is consistent with an adult-focused Delphi consensus statement published by the American College of Chest Physicians (ACCP) in 2001.[2] However, this strategy in children can result in long hospital stays due to persistent air leak, followed by high recurrence rates of 40 to 61\%.[3-9] Dissatisfaction with the outcomes of non-operative management and advent of minimally-invasive surgery has led some surgeons to offer primary video-assisted thoracoscopic surgery (VATS) for first episode PSP.[1,10] While it is not clear that primary VATS is the most cost-effective strategy, $[3,8]$ recent evidence suggests that an early decision to proceed to VATS after chest tube placement may be justified if we can predict which patients are likely to fail nonoperative management.[4,6,11]

Simple aspiration is recommended by the 2010 British Thoracic Society guidelines as first line intervention for PSP, in contrast to the ACCP chest tube first strategy.[12] A prospective randomized controlled study of simple aspiration versus chest tube drainage in adults suggested that aspiration was equally effective compared to chest tube drainage and avoided hospitalization in 50\% of patients with first PSP. A recent Cochrane Review also reported that aspiration is associated with shorter hospitalization and fewer adverse events, but may not be as effective as chest tube drainage in adults.[13] There is limited, low quality evidence around the use of simple aspiration in children, and this is not commonly used by North American pediatric 
surgeons.[1,11] Two recent retrospective studies in children suggest that simple aspiration is only successful in about $50 \%$ of children with PSP, less frequently than in adults.[14,15]

Given the lack of consensus around the initial management of pediatric PSP and suboptimal outcomes with the current common strategies, we sought to create a streamlined management pathway that would minimize the need for prolonged hospitalizations and reduce the incidence of recurrence. The purpose of this study was to evaluate the efficacy and role of simple aspiration for initial management of PSP in a pediatric population. Our hypothesis was that simple aspiration could prove useful as a test to predict which patients will fail non-operative management and would benefit from an early decision to proceed with surgical intervention.

\section{METHODS}

\section{Study Design}

We performed a prospective, non-randomized, multi-institutional pilot study investigating the role of simple aspiration in the initial management of children (12-17 years old) with primary spontaneous pneumothorax at nine children's hospitals in the Midwest Pediatric Surgery Consortium (MWPSC; www.mwpsc.org). The University of Wisconsin School of Medicine and Public Health served as the data coordinating center and the IRB of record (IRB \# 2014-1188). Since limited evidence exists in a dedicated pediatric population, this was designed as a pilot study to help guide future protocol development. Therefore, power calculation was not performed and the predetermined enrollment target was 33 patients. Subjects were enrolled from March 2016 through March 2018. Patients were eligible if 12 to 17 years of age, first presentation seeking medical attention for PSP, and the consulting pediatric surgeon determined that intervention was required for the condition. For a small pneumothorax $(<2 \mathrm{~cm}$ distance between chest wall and lung at the apex), a period of inpatient observation was allowed prior to 
decision for intervention. Exclusion criteria were recurrent pneumothorax, pneumothorax secondary to trauma or underlying pulmonary disease (i.e. cystic fibrosis, malignancy), bilateral pneumothorax, unstable patient with tension physiology, small pneumothorax $(<2 \mathrm{~cm})$ stable under observation, and chest drain placement at a referring facility. Consent and assent were obtained for data collection, including a follow-up phone survey at one year from enrollment.

Data were collected and managed using REDCap (Research Electronic Data Capture) software hosted in the Department of Surgery at University of Wisconsin-Madison. Protocol implementation and adherence monitoring was performed by a principle investigator at each site and bi-monthly study conference calls. Study compliance, safety reporting, and outcomes were reviewed by an independent data and safety monitoring committee at the primary site midway through enrollment.

\section{Simple aspiration protocol}

Figure 1

Aspiration was performed using a small catheter ( $\leq 12$ French) inserted into the pleural space under local anesthesia, with or without sedation, and with or without image-guidance, per local institutional practices. The study allowed flexibility in logistical details of the aspiration procedure, pragmatically allowing each site to leverage local strengths, established work-flows, and available resources. Suction was applied using either a syringe and 3-way stopcock or low suction tubing applied to the tube, with or without use of a waterseal controlled chest drain system. The tube was suctioned only transiently, just long enough to maximally expand the lung during the procedure, then the catheter capped or clamped immediately after suctioning. The catheter was maintained in place during an ensuing observation period of at least 6 hours, such that it could be utilized as a continuous chest drain should the aspiration test fail. 
Immediately after the aspiration procedure, a standard anterior-posterior projection chest radiograph (CXR) was obtained to evaluate and document lung expansion. The lung was considered adequately expanded if the lung apex reached the top of the $4^{\text {th }}$ rib or higher, and laterally the lung met the chest wall at the top of the $6^{\text {th }}$ rib or higher. If the lung was not adequately expanded, a second aspiration attempt was allowed using the same catheter. After aspiration and documenting adequate lung expansion, the patients were observed for at least 6 hours with the catheter capped, then another CXR obtained. If this film showed no pneumothorax or a small residual pneumothorax that was stable or smaller in size compared to the prior film, the catheter was removed and the patient discharged home. If the pneumothorax enlarged by radiographic or clinical signs at any time point after aspiration, or if the initial aspiration procedure did not adequately expand the lung or resolve symptoms, the patient was judged to have failed the simple aspiration test. Management after aspiration failure was per surgeon preference, not dictated by the study protocol. At surgeon discretion, such patients could be offered chest tube management (placing the existing catheter to suction) or immediate VATS. Patients with persistent air leak and prolonged chest drainage could proceed to VATS. All patients were followed with a return clinic visit at one month and a phone survey and medical record review one year from enrollment. 


\section{Statistical analysis}

Data summaries are expressed as means \pm standard deviation or proportions. Means between groups were compared using two-sample t-test and proportions compared using either Chisquare or Fisher's Exact tests. Time to recurrence was assessed with Kaplan-Meier methods and compared by log-rank test. Tests were considered significant if $\mathrm{P}<0.05$. Statistical analysis was performed using SAS 9.4 (SAS Institute Inc., Cary, NC).

\section{RESULTS}

\section{Simple aspiration outcomes}

A total of 33 subjects were enrolled and managed with the simple aspiration protocol. The number of subjects enrolled at each center was 10, 9, 5,3 (x3 sites), and 0 ( $\mathrm{x} 3$ sites). Sixteen of

Figure 2

$33(48.5 \%)$ passed the aspiration test, demonstrating adequate lung expansion that was stable after observation (Figure 2). Seventeen of $33(51.5 \%)$ failed the aspiration test and required further intervention due to evidence of ongoing air leak or residual pneumothorax, Of these 17, six $(35 \%)$ failed before completing 6 hours of observation. No statistically significant baseline differences were found between the group that passed aspiration versus the group that failed. (Table 1). Mean size of pneumothorax was similar between these two groups at presentation and after the aspiration procedure, but after observation the pneumothorax was significantly larger in the aspiration failure group, suggestive of ongoing air leak.

Of the 17 subjects who failed aspiration, 12 went on to non-operative chest tube management, with the existing catheter placed to suction, though two of those patients also required placement of a new chest drain. Five patients, at one institution, were managed by proceeding directly to immediate VATS per surgeon preference (Figure 2). 
No procedural complications occurred from the aspiration procedure itself. Of the 33 aspirations, 25 were performed without imaging, five with fluoroscopy, two with ultrasound guidance, and one procedure used both imaging modalities. Four subjects had a $2^{\text {nd }}$ attempt at aspiration for inadequate lung expansion on the first CXR after aspiration. Second aspiration resulted in PSP resolution in only 1 patient, and the other 3 went on to chest tube management.

\section{Simple aspiration as a predictor}

To evaluate the utility of simple aspiration as a test to predict need for VATS, incidence of recurrence and/or VATS were compared between the successful aspiration group versus the group that went to chest tube management following failed aspiration. This analysis excluded the five patients managed with immediate VATS after failed aspiration. The rate of recurrence and/or VATS in the chest tube management group was significantly higher than the recurrence rate after successful aspiration [10/12 (83\%) vs 7/16 (44\%), $\mathrm{P}=0.0028$, Table 2]. Of the 12 patients who failed aspiration and went on to chest tube management, 10 (83\%) ultimately required VATS - five during the initial hospitalization due to persistent air leak, and another five after outpatient recurrence. For the five that required VATS during the initial hospitalization, VATS was performed an average of 4.6 days after the initial aspiration procedure, suggesting that these patients did have an adequate trial of chest tube management before the decision for surgery. Two subjects who had a recurrence after non-operative management were treated nonoperatively once again; one in the aspiration success group and one in the chest tube group. Viewed as a predictive test, failure of simple aspiration predicted recurrence or need for operation with an $83 \%$ positive predictive value and $56 \%$ negative predictive value.

The time to event (either VATS or recurrence) for these two groups is shown as a KaplanFigure 3 Meier curve in Figure 3. Both curves demonstrate some early recurrences, including the 5 
patients who required VATS during the initial hospitalization, and both groups have some later recurrences as well. The earliest recurrence in the successful aspiration group occurred at 14 days, and the latest recurrence was in the chest tube management group at 379 days. The simple aspiration group was statistically less likely to experience VATS or recurrence at 380 days after initial hospitalization $(\mathrm{P}=0.028)$, further suggesting a prognostic benefit to the aspiration test. All patients were followed out to one year, except for two subjects who were lost to follow-up after the one month clinic visit. Interestingly, those are the two subjects in the chest tube management group not known to have a recurrence, although electronic medical record review at one year did not find any return encounters for PSP at the initial treating facility.

\section{Length of stay}

As expected by the definition of these two groups, the successful aspiration group had a significantly shorter length of stay (LOS) than the chest tube management group for the initial hospitalization (Figure 4, Table 2). Two patients in the aspiration group had LOS unexpectedly over 48 hours; one due to pain, the other due to diabetes management. In the chest tube group, the 5 patients with persistent air leak necessitating VATS had the longest LOS. Regarding the 2 chest tube patients who have not had a known recurrence, one had a relatively short LOS, while one had LOS over 125 hours. The mean length of stay was shorter in the group of 5 patients treated with immediate VATS after failed aspiration, as compared to the chest tube management group, though this effect did not reach statistical significance due to small numbers and wide variance [mean $87.9 \pm 34.5$ vs $144.2 \pm 130.8$ hours, respectively, $\mathrm{P}=0.338$ ]. Figure 4 shows the clustering of shorter stays for those treated with immediate VATS after failed aspiration, suggesting that immediate VATS rather than chest tube drainage could help avoid the prolonged LOS seem in some chest tube patients. The immediate VATS group also demonstrated a trend 
toward fewer CXRs compared to the chest tube management group [mean $6.8 \pm 2.9$ vs $13.4 \pm 7.7$, respectively, $\mathrm{P}=0.085]$.

\section{VATS outcomes}

A total of 20 out of $33(60.6 \%)$ patients ultimately underwent VATS blebectomy, which was combined with mechanical pleurodesis/pleural abrasion in 18 of 20, while 2 had pleurectomy. The recurrence rate after a first VATS procedure was 3 of $20(15 \%)$. In comparison, the combined recurrence rate after successful non-operative management (either aspiration or chest tube) was 12 of $23(52 \%)$. A chest CT scan was obtained in 13 of $20(65 \%)$ prior to proceeding with VATS.

\section{DISCUSSION}

This study found that simple aspiration for the initial management of first episode PSP in children successfully resolved the episode in $48 \%$ of patients, and recurrence after successful aspiration was $44 \%$, similar to the recurrence rate of initial chest tube management in prior studies.[4,6-8] In contrast, the patients who failed aspiration and went on to chest tube management had a significantly longer hospital stay and $83 \%$ went on to either VATS or suffered recurrence. This suggests that initial aspiration is useful to risk-stratify patients with PSP and predict which patients will benefit from an earlier decision for VATS. The recurrence rate of $15 \%$ after VATS in this study is consistent with other published reports. [4,6-8]

Non-operative management with chest tube drainage results in recurrence rates over $50 \%$, and two recent studies have found that $63 \%$ to $80 \%$ of patients end up undergoing VATS for definitive treatment.[4,6] As a result, several authors have questioned this strategy and examined a role for primary VATS.[4,6-8,10] While associated with lower recurrence and shorter hospitalization, primary VATS for all patients has not been found to be a cost-effective strategy, 
since every patient would incur the expense of surgery.[3,6,8] In order to avoid prolonged hospitalizations, reduce recurrence and minimize costs, predictors are needed for earlier identification of patients unlikely to respond to non-operative management, such that earlier VATS may be implemented. A recent retrospective review of 46 patients suggested that presence of an air leak or inadequate lung expansion within 48 hours of chest tube placement predicts need for surgery, similar to our findings after simple aspiration.[4]

Based on these results, we propose a simplified management algorithm presented in Figure 5. Children with non-recurrent PSP who pass the simple aspiration test can avoid hospitalization altogether, while those who fail aspiration can move directly to VATS, given the high probability that it will eventually be required. While the intent of this study was to evaluate outcomes of simple aspiration, a chest drain was left in place during observation. This was intentionally incorporated in the study design to help gain surgeon acceptance and willingness to try a new technique and also to avoid a second procedure for patients who failed aspiration, anticipating chest tube management as the next step. Admittedly, this is a different approach than simple aspiration performed with an angiocatheter or a thoracentesis kit. If the proposed algorithm in Figure 5 is adopted, however, such simplified methods of performing the aspiration could be readily utilized. A chest drain would not be necessary at all, since the subsequent options after aspiration are either discharge or surgery, eliminating chest tube management altogether. Future prospective studies are warranted to validate this algorithm and to test the efficacy of other simple aspiration techniques without a chest drain.

This study is limited by small sample size, pilot study design with lack of randomization, and comparisons drawn between groups created based on outcomes. Since this was a pilot study, systematic tracking of all eligible/non-enrolled and non-eligible PSP patients was not performed, 
which may introduce some element of selection bias. Selection bias is likely limited, however, as all centers adopted simple aspiration as a standard-of-care option offered to all PSP patients. Only 4 patients known to be approached for the study opted for chest tube management instead. Furthermore, there were no differences in baseline characteristics between those who passed vs failed aspiration, suggesting a rather homogenous population with limited opportunity for selection bias. Enrollment was slower than anticipated, likely due to strict inclusion/exclusion criteria, which excluded patients with recurrent disease, those with chest tubes placed at a referring facility, and small PSP that could be observed. This study was performed at academic, tertiary care children's hospitals, and some centers enrolled more than others, so the findings may not be generalizable beyond those environments. Taking a pragmatic approach allowed for variability in techniques between centers, based on local resources and workflows. Such variability may increase generalizability, although it could also bias efficacy and does not provide clarity for the optimal technique in any given environment. Each site did have both aspiration successes and failures, however, so it does not appear that the technique of aspiration introduced a source of systematic bias.

We conclude that a simple aspiration test predicts need for surgery upon first presentation of PSP in children. Successful aspiration results in outcomes comparable to historical chest tube management. A management algorithm incorporating aspiration as a test supports a decision for early VATS for those who fail, while likely minimizing risk of recurrence, costs, and unnecessary operations. 
FIGURE LEGENDS:

Figure 1. Flow diagram of aspiration protocol.

Figure 2. Outcomes of simple aspiration.

Figure 3. Kaplan-Meier curves showing time to event (VATS or recurrence) for the successful aspiration group vs the chest tube management group. The chest tube management group includes the 5 patients that eventually failed chest tube management and underwent VATS during the initial hospitalization.

Figure 4. Dot-plot of initial hospitalization length of stay for the successful aspiration group and the two groups after failed aspiration: chest tube management and immediate VATS. In the aspiration and chest tube groups, solid circle represents a patient who had a recurrence, while open circle represents a patient who did not recur. Diamond represents a VATS that occurred during initial hospitalization.

Figure 5. Proposed algorithm for management of all patients with primary spontaneous pneumothorax (PSP). Patients with non-recurrent, first-presentation PSP are eligible for the simple aspiration test. Consider VATS for recurrent disease. Definitive decision for discharge or VATS should include discussion of relative recurrence risks with patient/family. 


\section{REFERENCES:}

[1] Williams K, Baumann L, Grabowski J, Lautz TB. Current Practice in the Management of Spontaneous Pneumothorax in Children. J Laparoendosc Adv Surg Tech A 2018. [Epub ahead of print, as of 3.15.19] doi:10.1089/lap.2018.0629.

[2] Baumann MH, Strange C, Heffner JE, Light R, Kirby TJ, Klein J, et al. Management of Spontaneous Pneumothorax. Chest 2001;119:590-602. doi:10.1378/chest.119.2.590.

[3] Cook CH, Melvin WS, Groner JI, Allen E, King DR. A cost-effective thoracoscopic treatment strategy for pediatric spontaneous pneumothorax. Surg Endosc 1999;13:1208-10. doi:10.1007/PL00009622.

[4] Williams K, Lautz TB, Leon AH, Oyetunji TA. Optimal timing of video-assisted thoracoscopic surgery for primary spontaneous pneumothorax in children. J Pediatr Surg 2018;53:1858-61. doi:10.1016/j.jpedsurg.2017.11.047.

[5] Young Choi S, Beom Park C, Wha Song S, Hwan Kim Y, Cheol Jeong S, Soo Kim K, et al. What Factors Predict Recurrence after an Initial Episode of Primary Spontaneous Pneumothorax in Children? Ann Thorac Cardiovasc Surg 2014;20:961-7. doi:10.5761/atcs.oa.13-00142.

[6] Lopez ME, Fallon SC, Lee TC, Rodriguez JR, Brandt ML, Mazziotti MV. Management of the pediatric spontaneous pneumothorax: is primary surgery the treatment of choice? Am J Surg 2014;208:571-6. doi:10.1016/j.amjsurg.2014.06.009.

[7] Soler LM, Raymond SL, Larson SD, Taylor JA, Islam S. Initial primary spontaneous pneumothorax in children and adolescents: Operate or wait? J Pediatr Surg 2018;53:19603. doi:10.1016/j.jpedsurg.2017.12.014. 
[8] Qureshi FG, Sandulache VC, Richardson W, Ergun O, Ford HR, Hackam DJ. Primary vs delayed surgery for spontaneous pneumothorax in children: which is better? J Pediatr Surg 2005;40:166-9. doi:10.1016/j.jpedsurg.2004.09.042.

[9] Butterworth SA, Blair GK, LeBlanc JG, Skarsgard ED. An open and shut case for early VATS treatment of primary spontaneous pneumothorax in children. Can J Surg J Can Chir 2007;50:171-4.

[10] Chambers A, Scarci M. In patients with first-episode primary spontaneous pneumothorax is video-assisted thoracoscopic surgery superior to tube thoracostomy alone in terms of time to resolution of pneumothorax and incidence of recurrence? Interact Cardiovasc Thorac Surg 2009;9:1003-8. doi:10.1510/icvts.2009.216473.

[11] Williams K, Oyetunji TA, Hsuing G, Hendrickson RJ, Lautz TB. Spontaneous Pneumothorax in Children: National Management Strategies and Outcomes. J Laparoendosc Adv Surg Tech A 2018;28:218-22. doi:10.1089/lap.2017.0467.

[12] MacDuff A, Arnold A, Harvey J, on behalf of the BTS Pleural Disease Guideline Group. Management of spontaneous pneumothorax: British Thoracic Society pleural disease guideline 2010. Thorax 2010;65:ii18-31. doi:10.1136/thx.2010.136986.

[13] Carson-Chahhoud KV, Wakai A, van Agteren JE, Smith BJ, McCabe G, Brinn MP, et al. Simple aspiration versus intercostal tube drainage for primary spontaneous pneumothorax in adults. Cochrane Database Syst Rev 2017;9:CD004479. doi:10.1002/14651858.CD004479.pub3.

[14] Robinson PD, Blackburn C, Babl FE, Gamage L, Schutz J, Nogajski R, et al. Management of paediatric spontaneous pneumothorax: a multicentre retrospective case series. Arch Dis Child 2015;100:918-23. doi:10.1136/archdischild-2014-306696. 
[15] Soccorso G, Anbarasan R, Singh M, Lindley RM, Marven SS, Parikh DH. Management of large primary spontaneous pneumothorax in children: radiological guidance, surgical intervention and proposed guideline. Pediatr Surg Int 2015;31:1139-44. doi:10.1007/s00383-015-3787-8. 
Table 1. Comparison of baseline characteristics and post-aspiration pneumothorax size between subjects who passed vs failed the simple aspiration test

\begin{tabular}{|c|c|c|c|}
\hline & $\begin{array}{c}\text { Aspiration Pass } \\
(n=16)\end{array}$ & $\begin{array}{c}\text { Aspiration Fail } \\
(\mathbf{n}=17)\end{array}$ & P-value \\
\hline Male Gender & $12(75 \%)$ & $14(82 \%)$ & 0.688 \\
\hline Age (years \pm SDD) & $15.7( \pm 1.40)$ & $16.0( \pm 1.17)$ & 0.491 \\
\hline BMI $( \pm$ SD $)$ & $19.7( \pm 5.18)$ & $19.6( \pm 2.23)$ & 0.901 \\
\hline $\begin{array}{c}\text { Duration of symptoms } \\
\quad(\text { hours } \pm \text { SD })\end{array}$ & $33.3( \pm 25.15)$ & $18.6( \pm 18.7)$ & 0.138 \\
\hline $\begin{array}{l}\text { PTX size at presentation } \\
(\mathrm{cm} \pm \mathrm{SD})^{*}\end{array}$ & $4.2( \pm 1.32)$ & $4.0( \pm 1.57)$ & 0.641 \\
\hline $\begin{array}{l}\text { PTX size after aspiration } \\
(\mathbf{c m} \pm \mathrm{SD})^{*}\end{array}$ & $1.28( \pm 1.05)$ & $2.1( \pm 1.95)$ & 0.141 \\
\hline $\begin{array}{c}\text { PTX size after } 6 \text { hrs } \\
\text { observation }(\mathrm{cm} \pm \mathrm{SD})^{*}\end{array}$ & $0.66( \pm 0.58)$ & $2.3( \pm 1.61)$ & 0.0005 \\
\hline
\end{tabular}

*PTX = pneumothorax; size measured as distance between lung and chest wall at apex 
Table 2: Incidence of recurrence and/or VATS between aspiration success group versus the chest tube management group.

\begin{tabular}{|c|c|c|c|}
\hline & $\begin{array}{c}\text { Aspiration success } \\
(\mathbf{n = 1 6})\end{array}$ & $\begin{array}{c}\text { CT management } \\
(\mathbf{n = 1 2})\end{array}$ & P-value \\
\hline Recurrence/VATS & $7 / 16(44 \%)$ & $10 / 12(83 \%)$ & $\mathbf{0 . 0 2 8}$ \\
\hline $\begin{array}{c}\text { Time to recurrence } \\
\text { (days } \pm \text { SD, range) }\end{array}$ & $60.0( \pm 66.0,14-189)$ & $* 184.6( \pm 137.9,31-379)$ & N/A \\
\hline Length of Stay (hours \pm SD) & $26.8( \pm 20.9)$ & $144.2( \pm 130.8)$ & $\mathbf{0 . 0 0 2}$ \\
\hline
\end{tabular}

*Time to recurrence for CT management group excludes subjects who had VATS during initial admission 
Aspiration with insertion small-bore catheter $(\leq 12 \mathrm{Fr})$

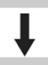

CXR to assess lung expansion

Observe for minimum 6 hrs with catheter capped

CXR to assess for recurrence

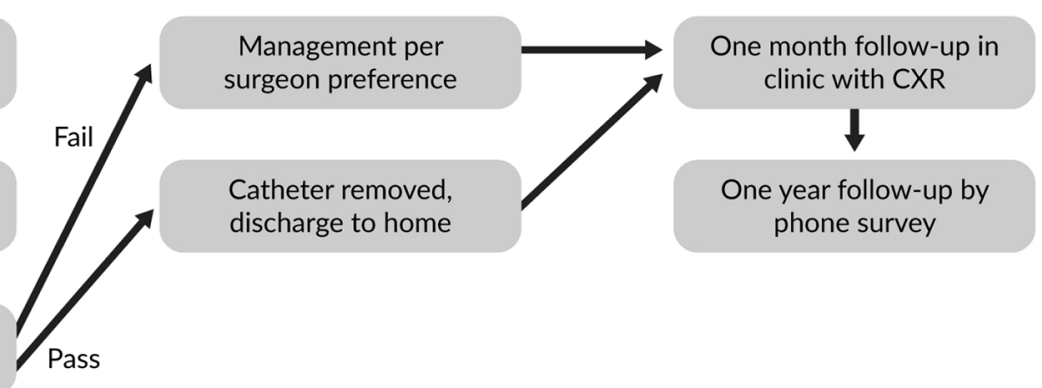

Figure 1 


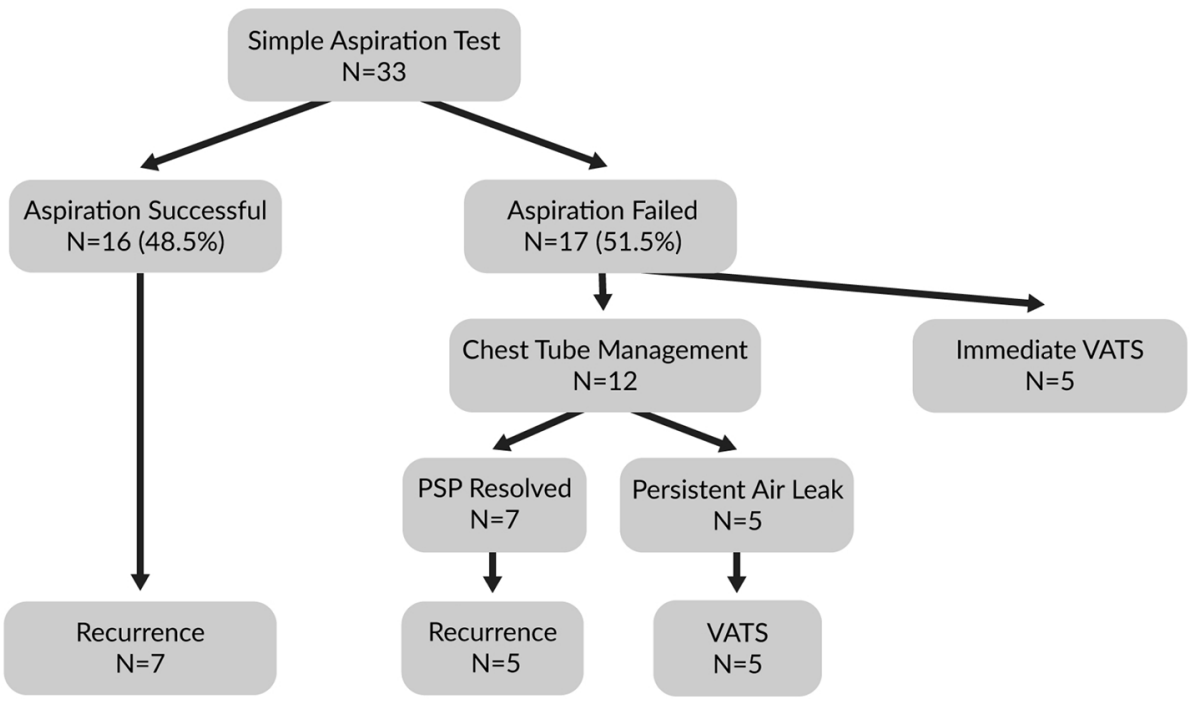

Figure 2 


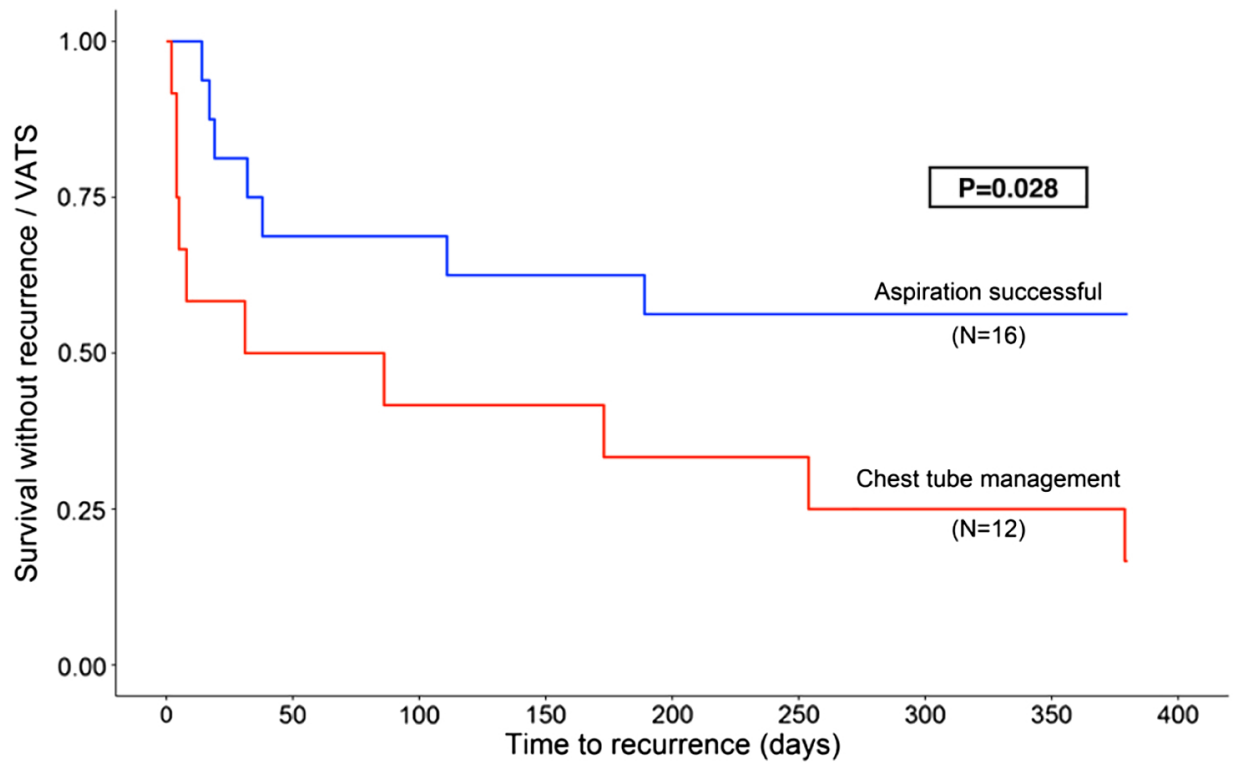

Figure 3 


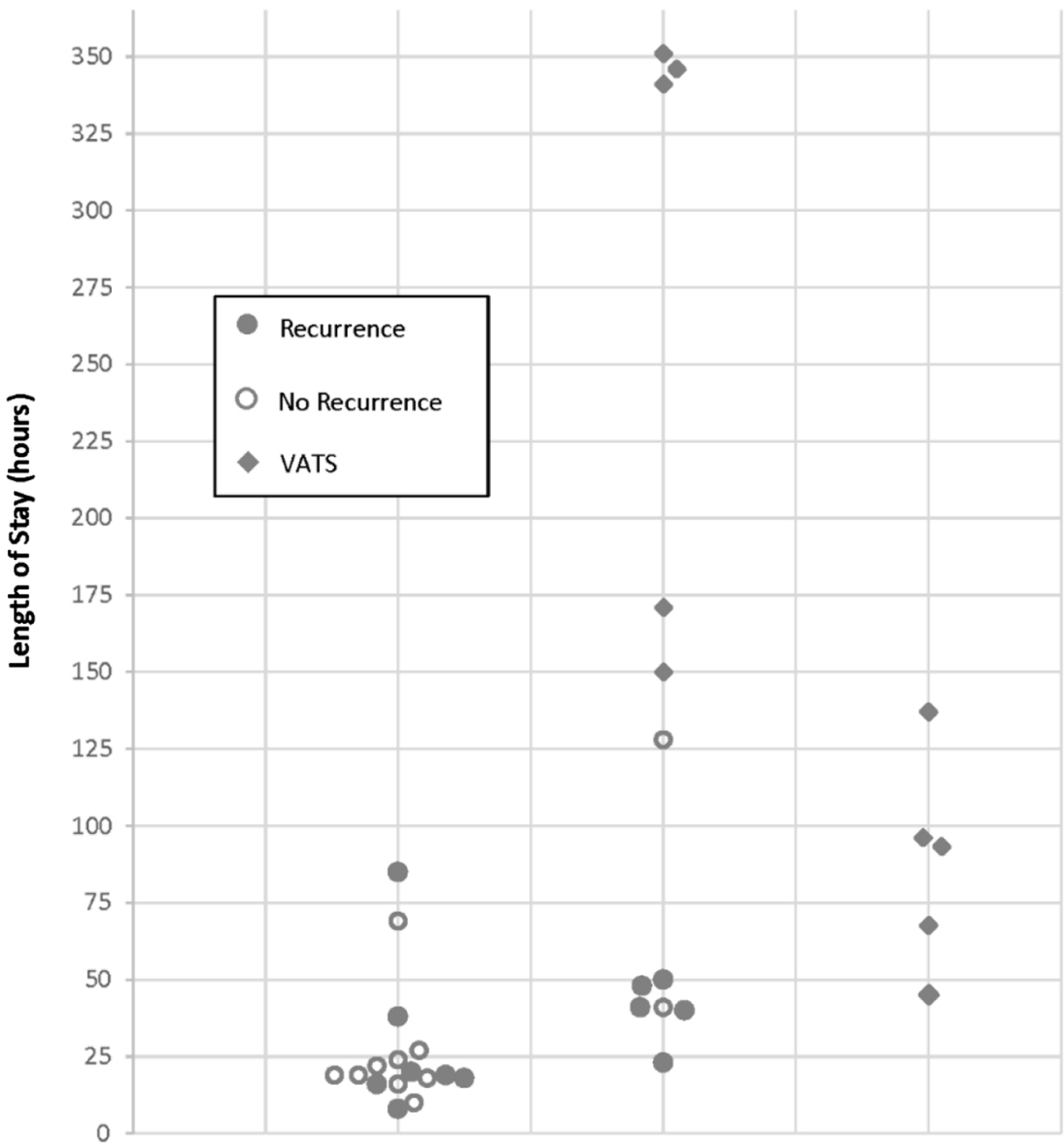

Successful

Aspiration

$n=16$
Chest Tube

Management

$n=12$
Immediate

VATS

$n=5$

Figure 4 


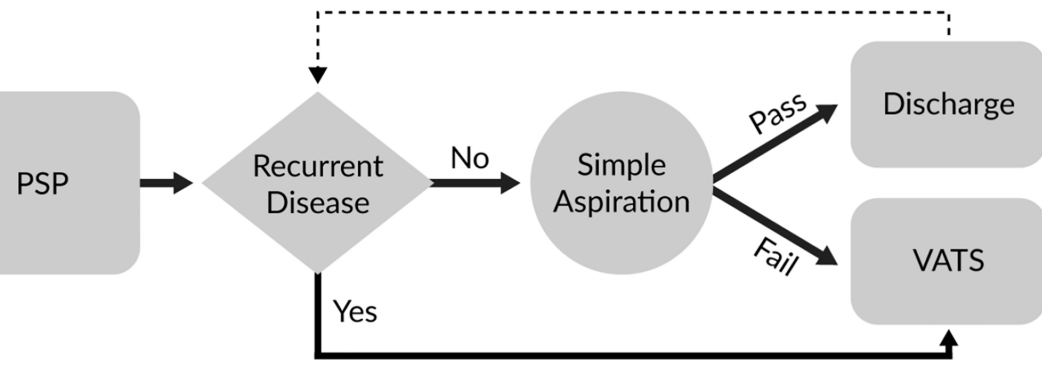

Figure 5 